\title{
Assistive technology to monitor activity, health and wellbeing in old age: The wrist wearable unit in the USEFIL project
}

\author{
V. Ahanathapillai ${ }^{\mathrm{a}}$, J.D. Amor ${ }^{\mathrm{b}, *}$ and C.J. James ${ }^{\mathrm{b}}$ \\ anstitute of Digital Healthcare, University of Warwick, Coventry, UK \\ ${ }^{\mathrm{b}}$ Warwick Engineering in Biomedicine, School of Engineering, University of Warwick, Coventry, UK
}

\begin{abstract}
This paper presents the assistive technology used to perform activity monitoring in the USEFIL (Unobtrusive Smart Environments for Independent Living) project, particularly the wrist wearable unit. USEFIL includes a number of activity monitoring devices alongside some condition specific medical devices, a dedicated electronic health record database and communication backend. The system is designed as an assistive technology to provide long-term monitoring for older people in their own home and communicate the data that is gathered into a decision support system that can be used by the older person's carers to improve their care and allow them to remain independent in their own home. The wrist wearable device developed for the USEFIL project, the various health indicators extracted from its inbuilt sensors and how these are used to understand the health and wellbeing of the older person are discussed in this paper.
\end{abstract}

Keywords: Assistive technology, unobtrusive monitoring, wrist wearable device, activity monitoring, old age

\section{Background}

Life expectancy continues to increase with the advancements in medicine, and also the rising awareness of the importance of health, nutrition and hygiene. Longer life expectancy results in an increase in the number of people with age related health conditions [1], i.e., there will be a greater number of old people in society, who might therefore need assistance in order to maintain their independence and daily activities. Furthermore, there is a decreasing ratio of caregivers to care-receivers and the growing constraint is that care must be provided with limited financial resources [2]. There is also a strong desire for older people to remain in their own home as they grow older, and for this to be supported in order to maintain their independence for as long as possible [3].

*Corresponding author: James Amor, School of Engineering, University of Warwick, Coventry CV4 7AL, UK. E-mail: J.D.Amor@ warwick.ac.uk.
In conjunction with the rise in life expectancy has been an ever increasing development of information and communication technology, with a corresponding improvement in performance and reduction in price. Technology is widely used to develop solutions in situations where resources are a constraining factor and emerging healthcare needs present a problem that seems suited to a technological solution, at least in part. There is an opportunity to use and influence the development of technology to provide affordable and scalable healthcare [4]. Through using appropriate technologies, healthcare systems can be delivered and can provide effective healthcare within emerging resource constraints. More often, especially for providing long term care or management, the solutions that emerge have some technological component and activity monitoring is one of a range of emerging technologies with applications in this area.

\subsection{Activity monitoring}

Activity monitoring is the process of monitoring a person's activity and behavioural pattern over a period 
of time and using the data collected from this process to aid in their care. As the activity of a person is related to their health, assessing the activity levels continuously is essential in order to recognize a change in health status [5]. Also, in order to meet the amount of moderate and vigorous physical activity recommended for older adults by the World Health Organization, (WHO) [6], a quantitative or direct measure for activity level is preferred in comparison to self-reported measures [7]8].

A person's activity can be monitored using a variety of sensors, which can be broadly classified as environmental or wearable. Environmental sensors provide information about the behaviour of the user within their home/work environment. Examples of environmental sensors include door/window switches, motion detectors (also known as room occupancy sensors) such as passive infra-red (PIR) sensors, motion capture systems (video/camera systems, Kinect), power consumption monitors and also sensors to measure the physical aspects of the environment such as light, temperature, sound and pressure sensors. By using a combination of these sensors, data can be collected which describes the activity of the user within their environment [9].

Wearable sensors or body worn sensors provide information on the activity of the user directly. The wearable sensors include kinematic sensors or accelerometers, GPS (location) sensors, and temperature, pressure, light and camera sensors. Accelerometers are widely used for the purpose of monitoring activity and are used by placing a tri-axial accelerometer on the chest [1011], waist [12,-15] or multiple locations [16, 17]. The general considerations for choosing an accelerometer for use in studies with older adults is given by [18] to include the lifestyle PA, step counts and energy estimation. Though, having information from various wearable sensors can give a very good picture of the person's activity, a simple system using sensors attached to the wrist is generally preferred, especially for an older person [19].

The application of activity monitoring is widespread and varied, ranging from general well-being to rehabilitation. Activity monitoring has its applications in the general wellbeing of any person, active living, sports, exercise and monitoring daily activities. It can be beneficial to monitor activity when managing diabetes and obesity, long-term chronic conditions such as chronic obstructive pulmonary disease (COPD) [20] and stroke rehabilitation [21]. Monitoring activity is advantageous in studying conditions such as chronic fatigue syndrome and fibromyalgia [22]. In old age, it is particularly beneficial to monitor the functional ability of a person to perform Activities of Daily Living (ADL) [23], which is advantageous in monitoring independent living in older people.

This paper introduces the European Union $7^{\text {th }}$ framework programme funded, Unobtrusive Smart Environments For Independent Living (USEFIL) project [24] and presents the wrist wearable unit (WWU) that has been developed as part of the USEFIL project. This paper outlines how activity monitoring can be applied to independent living and how the WWU has been developed to provide an ambulatory monitoring platform. Specifically, this paper presents monitoring parameters extracted by the WWU and the validation process that has been used to test these in normal controls prior to further trials with different target groups.

\section{Objective}

The aim of the USEFIL project is to develop assistive technology for older people using low cost, offthe-shelf devices to provide affordable in-home unobtrusive monitoring and web communications in order to promote independent living, feelings of security and social connectivity. Within USEFIL, the aim of the WWU is to provide an unobtrusive monitoring platform that can monitor parameters relating to physical activity and health and integrate with the wider USEFIL system.

\section{Methods}

The USEFIL system includes a number of activity monitoring devices alongside some condition specific medical devices and a dedicated electronic health record (EHR) and communication backend. The system is designed to provide long-term monitoring for older people in their own home and feed the data that is gathered into a decision support system (DSS) that can be used by the older person's carers to improve their care and allow them to remain independent in their own home. The overall USEFIL system is presented in Fig. 1, showing the technology that is available for the older person in the home environment and the USEFIL server where the data is transferred to be analysed for long term trends, this will also send alerts to the carer/health care professionals, who are connected to the system via the internet.

The different technology or devices used in the USEFIL system are given below: 


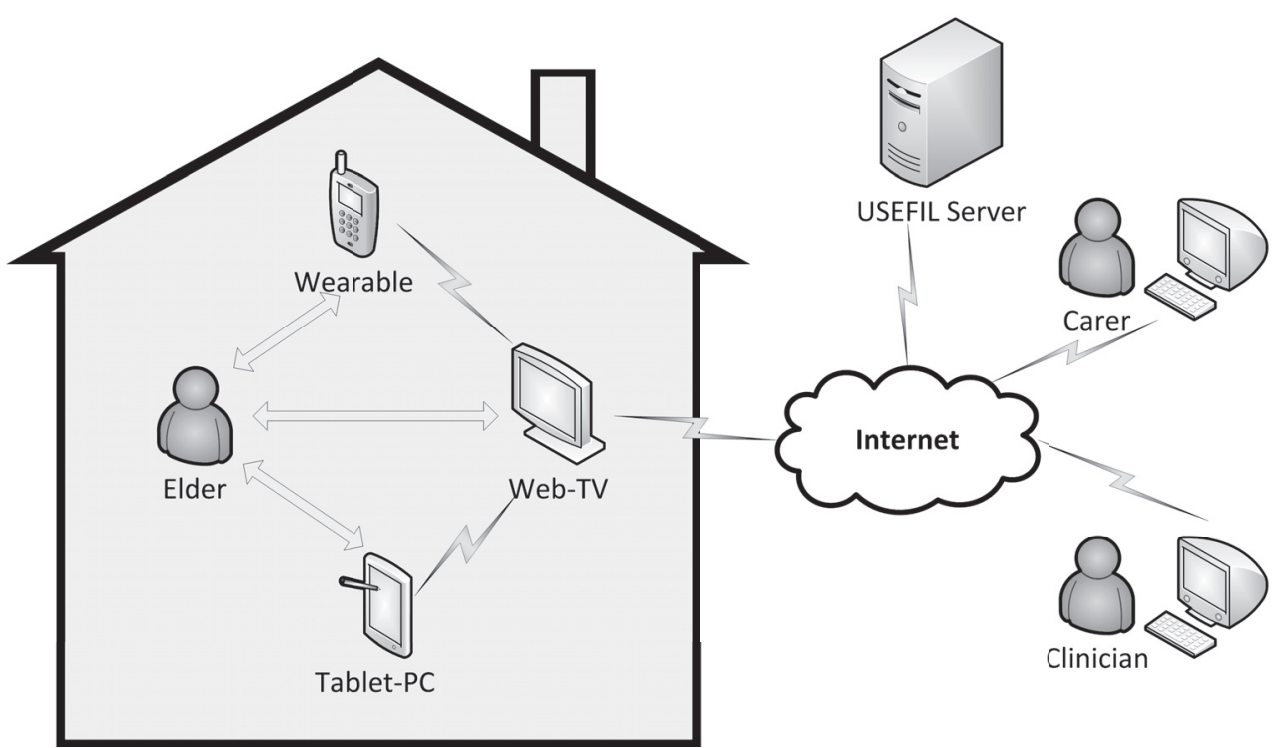

Fig. 1. The overall USEFIL system showing the technology that is available for the older person in the home environment and the USEFIL server where the data is transferred to be analysed for long term trend to alert. The carer and the health care professional are connected to the system via internet.

- Slate Tablet PC: Provides connection to a social network for older users. The bigger screens showing smartphone apps much easier for the older person to use them. It helps to observe the social connectivity of the person and detect social isolation.

- Web-TV: Provides access to TV channels, personalisation, games, social networking etc. To deliver content from other computers or storage devices. It helps to observe the social connectivity of the person and detect social isolation. The games available in the TV provide notifications on cognitive decline.

- Kinect: To monitor the balance or stability of the older person and monitor some ADL.

- A low cost camera embedded in a mirror to monitor vital signs such as heart rate and breathing rate, for long term monitoring. This also helps in detecting changes in emotions which could indicate health problems.

- WWU: Acts as a multi-sensor data logging device to record activity, monitor ADL, detect activity related parameters and vital signs, in order to infer behaviour patterns. This is useful for continuous long term monitoring of the person.

Along with these technologies available to the user, there are few core components and services that work in the background in order to provide essential system services.
- EHR: To store the sensor data from these devices in EHR database in a server.

- DSS: To process all the sensor information to infer the well-being of the person, for example to look at the trend of a particular health indicator and send alerts to the health professional to have a look at the data and intervene to change a medication or arrange for a check-up, etc. The DSS also has various useful applications, including application to send reminders to take medications, to remind appointments or important dates in their calendar, to send recommendations to the older person to more activity based on the DSS inference.

- Web-interface: For the health professional/carer to access the patient data on the trend in

- Emergency alert: This enables the older person to inform their selected contacts, if an emergency occurs.

\subsection{The wrist wearable unit}

There are a number of devices available on the market that are either specifically designed for health based monitoring (including devices such as Actigraph [25], Fitbit [26] and Jawbone [27]), or that can be adapted to this use. Of these devices, smart-watches best suit the needs of the USEFIL system, as the system requires an open device that can connect and communicate with the rest of the off-the-shelf devices in the 
Table 1

Z1 Device specifications

\begin{tabular}{ll}
\hline Device name & Z1 Android Watch-Phone \\
\hline Form factor & Watch \\
Dimension & $64 \mathrm{~mm} \times 42 \mathrm{~mm} \times 14.5 \mathrm{~mm}$ \\
Weight & $160 \mathrm{~g}$ \\
Operating system & Android 2.2 \\
Processor & MediaTek MT6516 @ 416 MHz \\
Memory & $256 \mathrm{MB}$ RAM \\
Removable storage & $8 \mathrm{~GB}($ Micro SD) \\
Connectivity & USB, Bluetooth and Wi-Fi \\
Battery & $800 \mathrm{mAh}$ \\
Camera & $3.0-5.0 \mathrm{M}$ \\
Screen & 2.0 inch, 320 $\times 240$ pixels \\
& TFT displaying and multi-points touch screen \\
Frequency ranges & GSM850/900/1,800 and 1,900(GPRS), SIM \\
\hline
\end{tabular}

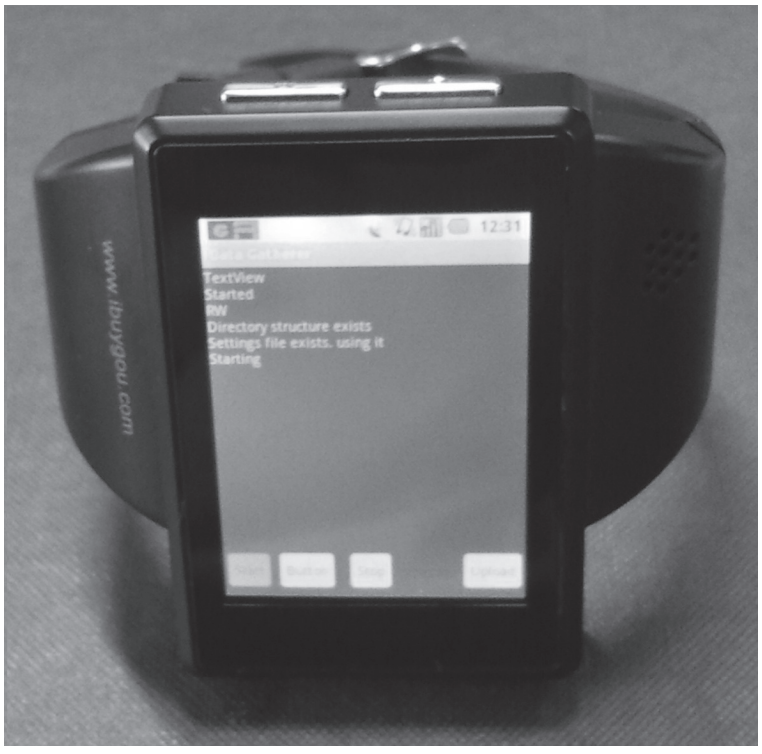

Fig. 2. The Z1 smartwatch used in the USEFIL project to monitor activity.

system. Smartwatches combine some integrated sensors with the computational power and communication capabilities that are required for the USEFIL project. Of the available smartwatches on the market, Android based devices best fit the specification and were chosen as the target platform, in part because an App could be developed and continually moved to newer, better devices as they became available.

The device that was chosen for the USEFIL project at the time of writing was the Z1 Watch Phone [28], shown in Fig. 2, which is a fully featured Android 2.2 smartphone in a wrist wearable form factor. The Z1 has a tri-axis accelerometer (with sampling rate options of $5 \mathrm{~Hz}, 16 \mathrm{~Hz}$ and $50 \mathrm{~Hz}$, and a g-range of $\pm 2 \mathrm{~g}$ ), camera and GPS receiver as well as full suite of processor, memory and communications options (including
GSM and Wi-Fi) that are standard for smart-phones. The processor on the $\mathrm{Z} 1$ is sufficient to allow for multitasking, enabling the $\mathrm{Z} 1$ to perform background data gathering tasks at the same time as foreground user interaction tasks. The detailed device specification of $\mathrm{Z} 1$ is provided in Table 1 . The data processing in the WWU that extracts the activity monitoring parameters have been validated with several test groups of normal controls to validate the general functionality of the algorithms, aid their development and fix any early stage issues. This has been carried out prior to testing with different target groups of older people as it is desirable to minimise the amount of repeat testing being carried out by potentially vulnerable user groups.

\section{Results}

The USEFIL project's ambulatory monitoring is performed by using the inbuilt tri-axis accelerometer sensor in the WWU. The raw tri-axis accelerometer reading showing $\mathrm{X}, \mathrm{Y}$ and $\mathrm{Z}$ axis data and their root mean square combination are presented in Fig. 3. The activity related parameters extracted from such a raw accelerometer signal are the activity levels of the person, step counts, and device worn status. This section presents the results obtained for the various activity related parameters extracted from accelerometer and the heart rate extracted from the inbuilt camera.

\subsection{Activity levels}

The root mean square (RMS) of the acceleration data increases as the activity level increases, meaning that the use of an RMS score for a measure of activity is valid [29]. In [30], it is shown that the vector magnitude of the tri-axis accelerometers correlates 

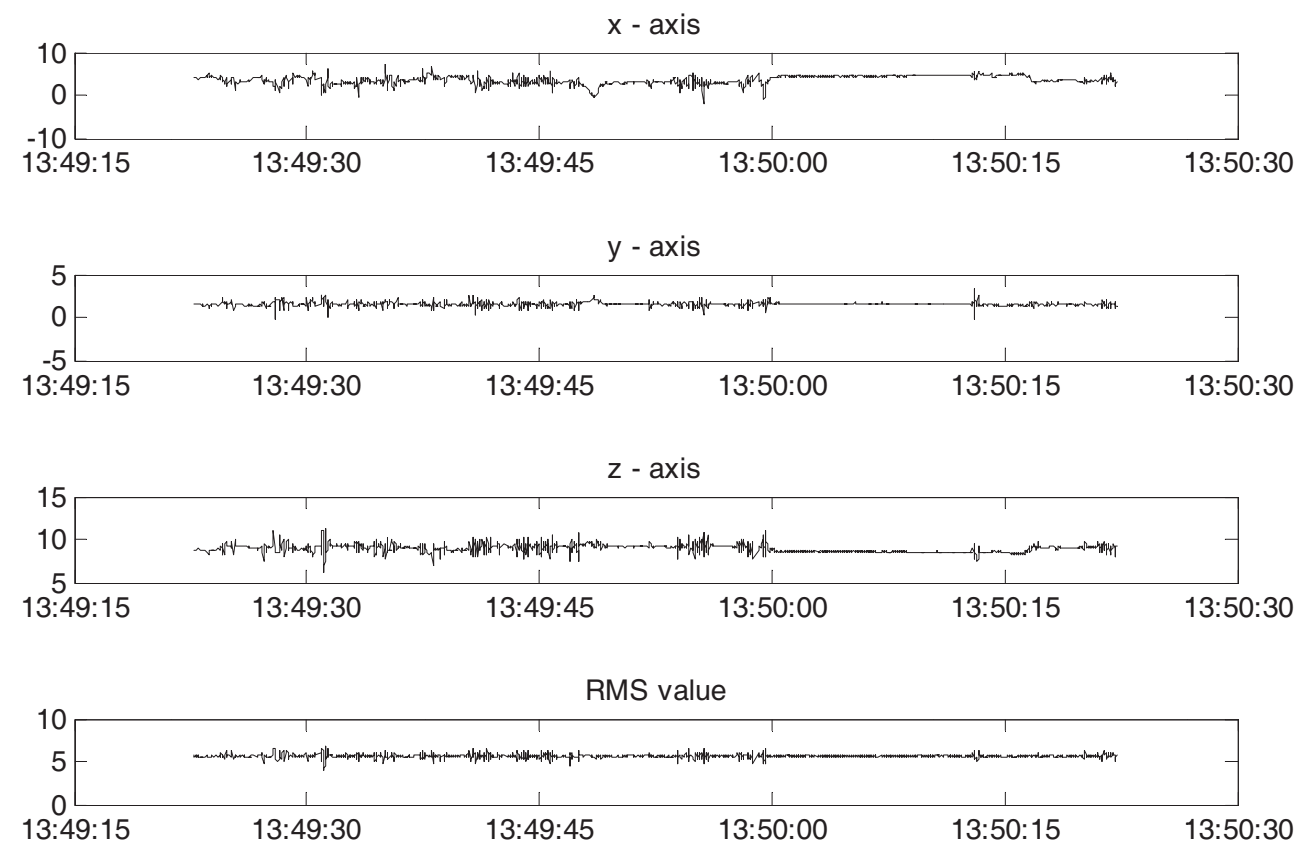

Fig. 3. Raw accelerometer signal showing $\mathrm{x}, \mathrm{y}, \mathrm{z}$ axis data and the root mean square combination.

(a) RMS combination of raw $\mathrm{X}, \mathrm{Y}$ and $\mathrm{Z}$ axis accelerometer data

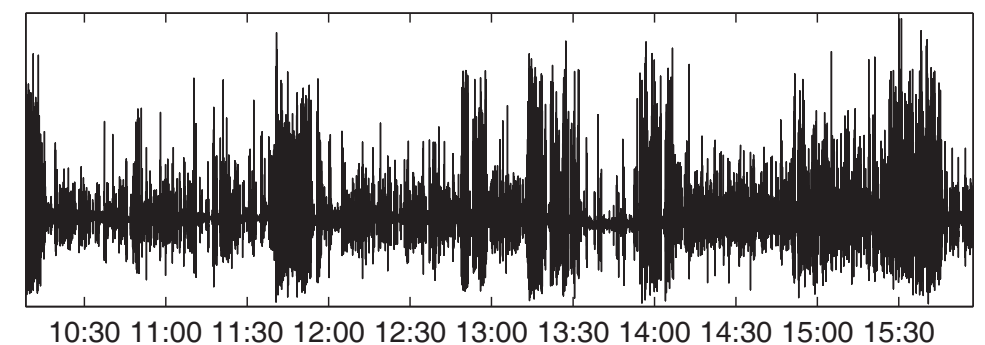

(b) Activity Bar

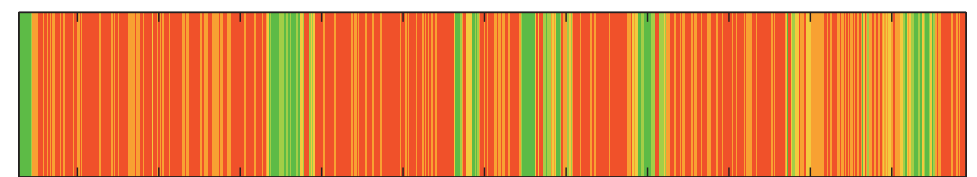

10:30 11:00 11:30 12:00 12:30 13:00 13:30 14:00 14:30 15:00 15:30

Very Low Low Hedium High Very High

Fig. 4. (a) Root mean square (RMS) combination of the X, Y and Z axis accelerometer data and (b)Activity bar obtained by processing the RMS combination. (Colours are visible in the online version of the article; http://dx.doi.org/10.3233/TAD-150425)

with the energy expenditure for a range of activities. The RMS combination of the raw $\mathrm{X}, \mathrm{Y}$ and $\mathrm{Z}$ axis accelerometer data (as shown in Fig. 4(a)) is used to determine the activity levels. The RMS data is split into non-overlapping windows of length $\mathrm{N}$. The local mean in the window is subtracted and then the average of the absolute values is taken. This represents the activity score and is converted into an activity level by comparison with a set of thresholds as follows.

1. Very low activity (value $<$ threshold 0 ) 


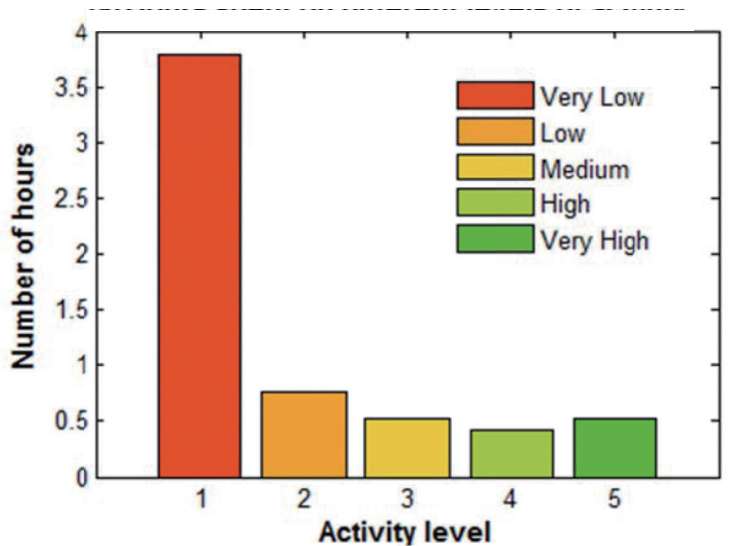

(a) Hours spent on different levels of activity

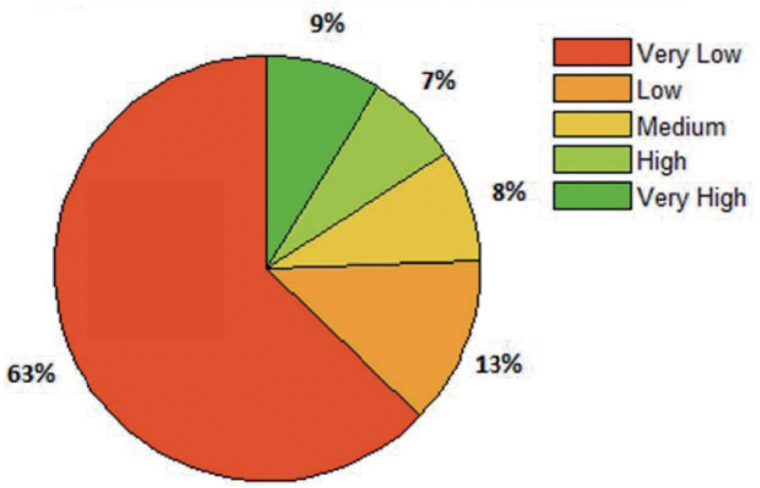

(b) Percentage of time spent of different activity levels

Fig. 5. (a) Bar graph showing the time spent by the person in the entire data set performing the different levels of activity (here it is hours spent). (b) Pie chart showing the percentage of total time spent by the person performing different levels of activity. (Colours are visible in the online version of the article; http://dx.doi.org/10.3233/ TAD-150425)

\section{Low activity}

(threshold $0<$ value $<$ threshold 1 )

3. Medium activity

(threshold $1<$ value $<$ threshold 2 )

4. High activity

(threshold $2<$ value $<$ threshold 3 )

5. Very high activity

(threshold $3<$ value)

The thresholds used here are based on the maximum value of the activity scores generated from the data. This value corresponds to the highest level of activity of the person. Further details on the activity levels algorithm can be found in [31]. Each activity score is given a colour coding (very low activity - red, low activity - orange, medium activity - yellow, high activity - light green, very high activity - green). This colour coded data set is shown as an activity bar, and is plotted for easy interpretation.
Figure 4(b) shows the activity bar obtained by processing the RMS combination. The activity bar provides a visual interpretation of when (what time of the day) the person was more active and when the person was less active. When the activity level is monitored over time, it is beneficial to understand the activity routine of the person. For example, it can be displayed in the day-by-day format which makes it easier to visualise the activity pattern (active and resting period) during a day. Building it up over weeks/months could identify the activity pattern of the person during the day. Once this baseline activity pattern is identified for a person, any deviation from this pattern can be detected automatically. Any anomaly in the activity could provide information on the health status of the person. For example, if the person is less active than usual, then this may be a case when the person is not well, and the system can generate an automatic alert.

The activity levels extracted from the raw data can also be represented as an activity breakdown, showing the percentage of time spent on different levels of activity. This can be presented in a pie chart or a bar graph. Figure 5(a) gives a bar graph showing the time spent by the person in the entire data set performing the different levels of activity (shown in hours spent) and (b) gives a pie chart showing the percentage of total time spent by the person performing different levels of activity (with the appropriate colour coding). This is beneficial to understand the overall activity level of the person.

Activity level calculations have been verified empirically in a small group of users $(n=3)$ through long term usage of the device. Participants were asked to wear the device for several days and keep a rough $\log$ of their behaviours and actions. These were cross validated against the recorded activity levels and also against recorded step-counts. They were found to be well correlated for all participants.

\subsection{Step counts}

Figure 6 shows the tri axis accelerometer data of a walking pattern recorded from a healthy control. It can be seen that the walking produces a rhythmic pattern in the accelerometer. The step counts can be obtained from the RMS combination of the tri-axis accelerometers. Step count detection is performed by extracting the walking frequency (number of steps per second) from the data. This can be obtained by applying a signal processing technique called Fast Fourier Transform (FFT), which looks at the frequency content of the sig- 


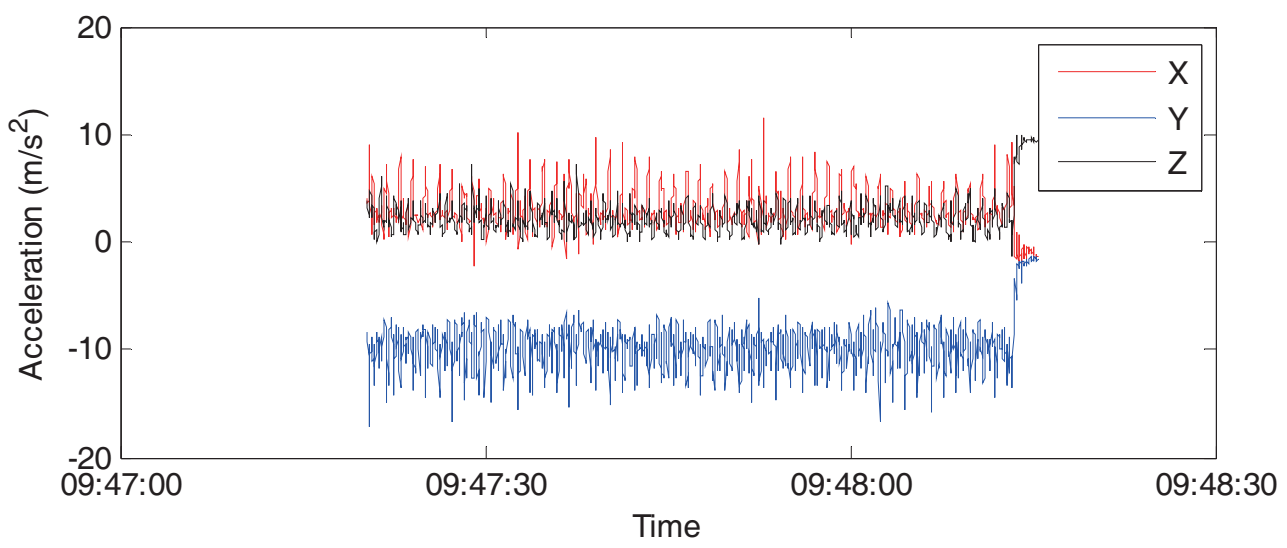

(a) Raw acceleration data

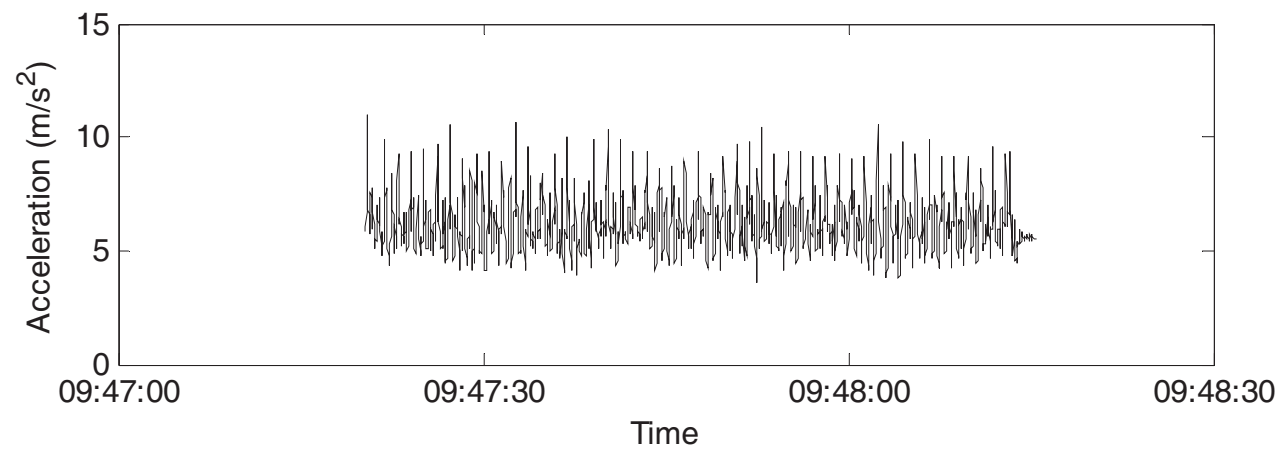

(b) RMS value

Fig. 6. Walking pattern (a) Raw acceleration signal and (b) the RMS combination. (Colours are visible in the online version of the article; http:// dx.doi.org/10.3233/TAD-150425)

nal. The signal is reconstructed by inverse FFT, using a band of frequencies around the walking frequency and discarding the unwanted frequencies. Then the steps are calculated by identifying the peaks in the signal.

The step count algorithm is tested on the data collected using the WWU from 20 healthy participants ( 7 females and 13 males, age range: 20 to 50 years, heights: 160 to $191 \mathrm{~cm}$, and weights: 47 to $110 \mathrm{~kg}$ ). The WWU was worn on their non-dominant wrist, with the accelerometer sampling rate of $50 \mathrm{~Hz}$. The participants were asked to do the following activities:

1. Walking 100 steps at participant's normal pace

2. Walking 100 steps at a pace of $1 \mathrm{~Hz}$

3. Walking up stairs at participant's normal pace

4. Walking down the same stairs at participant's normal pace

These activities were chosen to give a range of walking types for analysis. The accelerometer data for normal walking, walking up stairs and walking downstairs are shown in Fig. 7. The $1 \mathrm{~Hz}$ pace was chosen to en- force a slow walking pace and also had the side effect of disrupting the participant's normal gait. For the stair sections the participants step count was manually recorded. The percentage error for each of the step counts is calculated to use for statistical analysis. A Two One-Sided Test (TOST) was performed to check if any of the sets of step counts were equivalent to the truth (percentage errors were statistically equivalent to $0)$. The equivalence to 0 was defined as being within $3 \%$ of 0 [32] and used a $95 \%$ confidence level.

Recording step counts is a useful activity related parameter which can help in monitoring the physical activity of a person. Identifying the number of steps the person has performed during the day can be useful as a motivating factor in order to increase physical activity and improve health [33]. It could also be used to set as a baseline for the person. A significant reduction in step count from the normal baseline of the person can also be used to generate alerts to the carer, in order to check on the person. As step count is a measure of physical activity, it can be used to show that physi- 


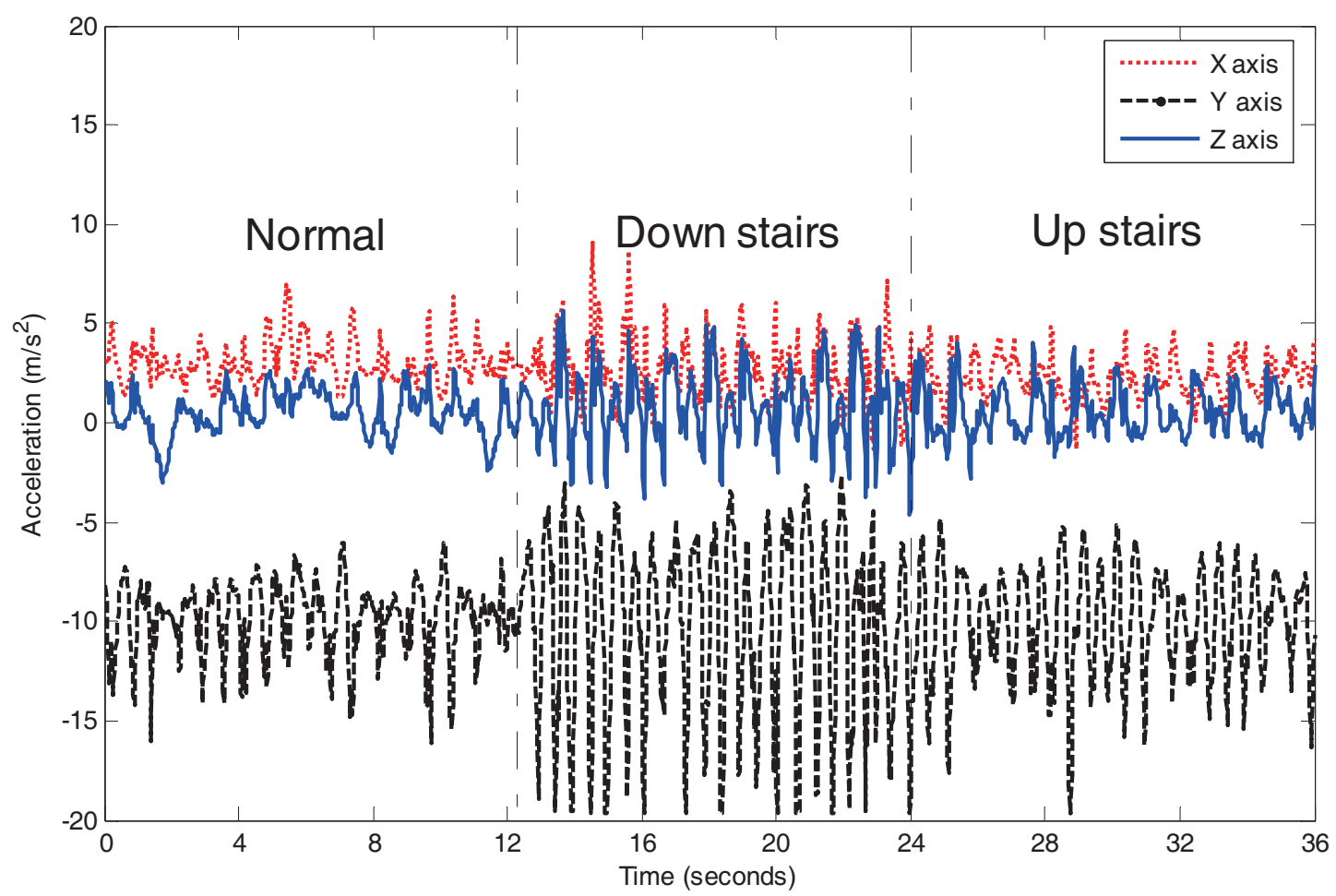

Fig. 7. Accelerometer data of three different walking patterns (normal walking, walking down the stair and walking up the stairs) for a single subject. (Colours are visible in the online version of the article; http://dx.doi.org/10.3233/TAD-150425)

cal activity can affect health outcomes, particularly in chronic conditions [34].

\subsection{Device worn status}

Detecting the worn status of the WWU is achieved by examining the accelerometery signal and by looking for the presence or absence of movement once the gravity component in the accelerometer signal is removed. It can be observed that when the device is not being worn the only force acting on the accelerometer is gravity. Once the gravity component is removed, if the device is not being worn, values close to zero will be detected.

The worn status detection has been empirically verified by testing the algorithm on different data-sets containing sections where the device was worn and not worn and observing the output. Figure 8 shows the results of the verification. Data were analysed in blocks of 1 minute duration. It can be shown from the figure that worn-state is correctly identified for all the test data. Sections that show an apparent overrun (where the worn-state indicates the device is being worn where it is not) are caused by the analysis of the data in $1 \mathrm{~min}$ blocks, where the user puts on or removes the WWU in the analysis block the algorithm determines that the WWU was worn.

\subsection{Heart rate}

The heart rate monitoring is performed in the WWU using the device's on-board camera. Figure 9 shows the heart rate processing from the raw camera movie which is processed frame by frame and then integrated to extract the heart rate. The fingertip is placed next to the camera and a 15 second movie is recorded. The change of volume in blood vessels causes a change in the amount of light absorbed by the fingertip, which correlates with heart rate. Thus, the subject's pulse rate is derived by analysing the recoded movie. Analysing the change in light of frames over time can therefore be used to find a pulse. Once the pulse for 15 seconds is calculated, it is multiplied by four to get the pulse rate per minute.

The heart rate detection on the WWU was tested for 10 healthy young subjects, which was then compared with the pulse oximeter CMS50E [35]. The heart rate of the subjects was measured using the heart rate detection application in a good ambient light and in parallel the pulse was measured using the CMS50E. The 


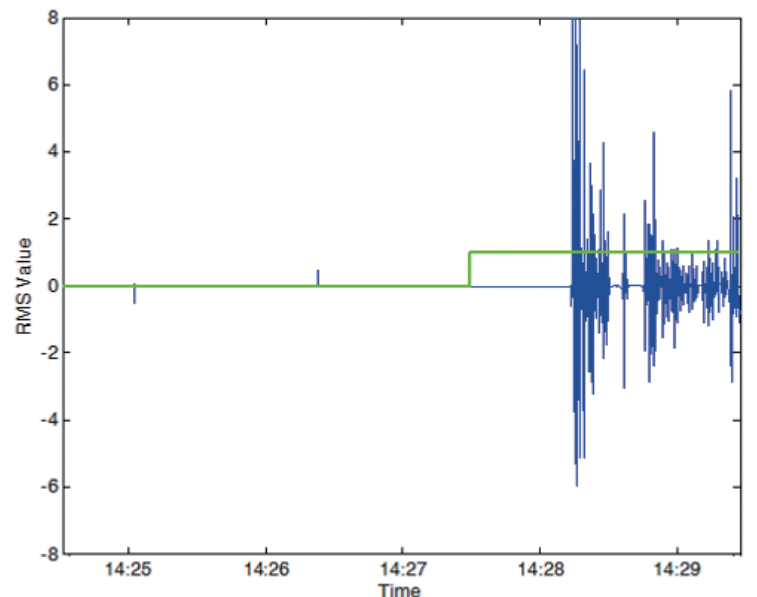

(a) Worn at the end of signal

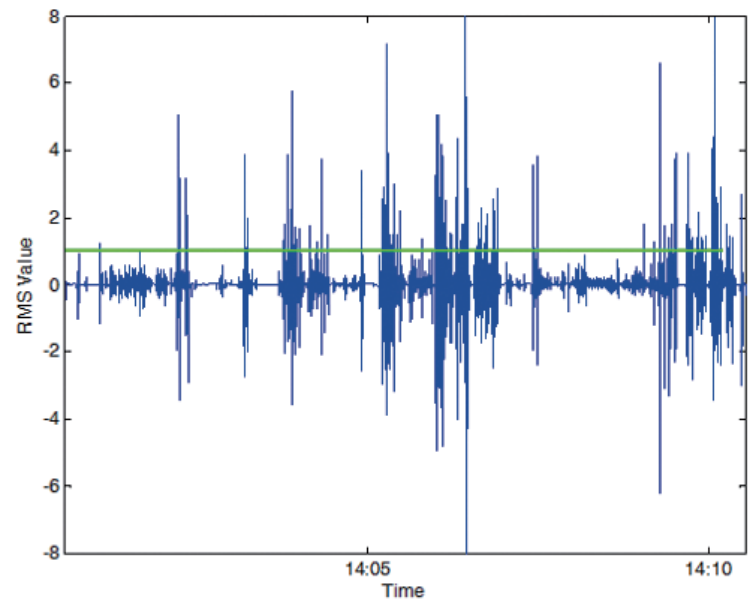

(c) Worn throughout

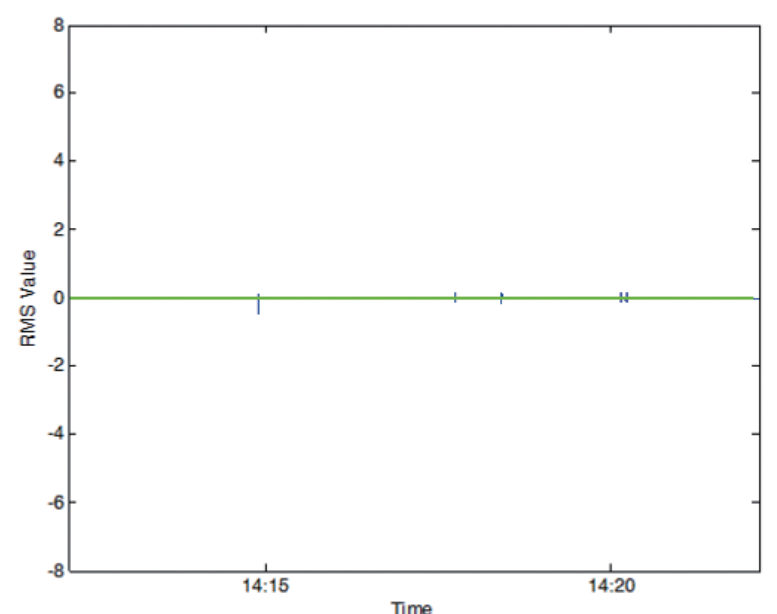

(b) Not worn throughout

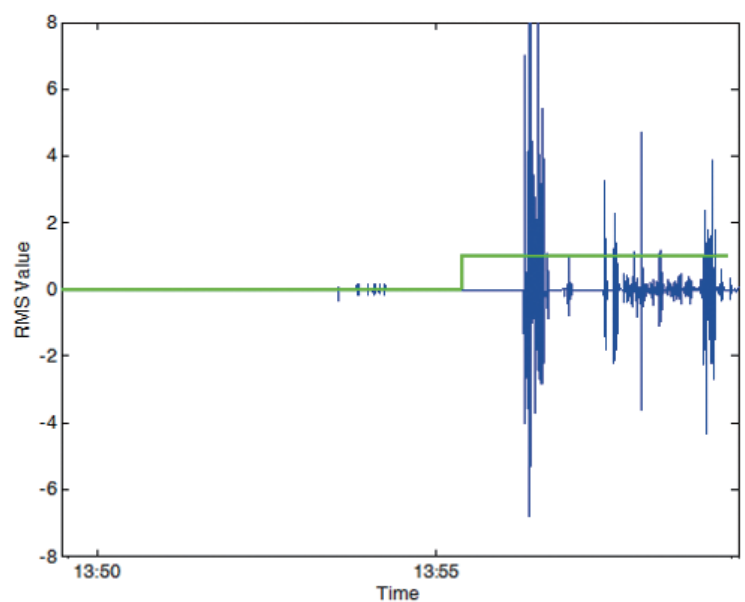

(d) Worn at the end of signal

Fig. 8. Device worn satus. RMS signal shown in blue; Worn status shown in green where 1 indicates worn and 0 indicates not worn. (Colours are visible in the online version of the article; http://dx.doi.org/10.3233/TAD-150425)

\section{Raw Camera Data}

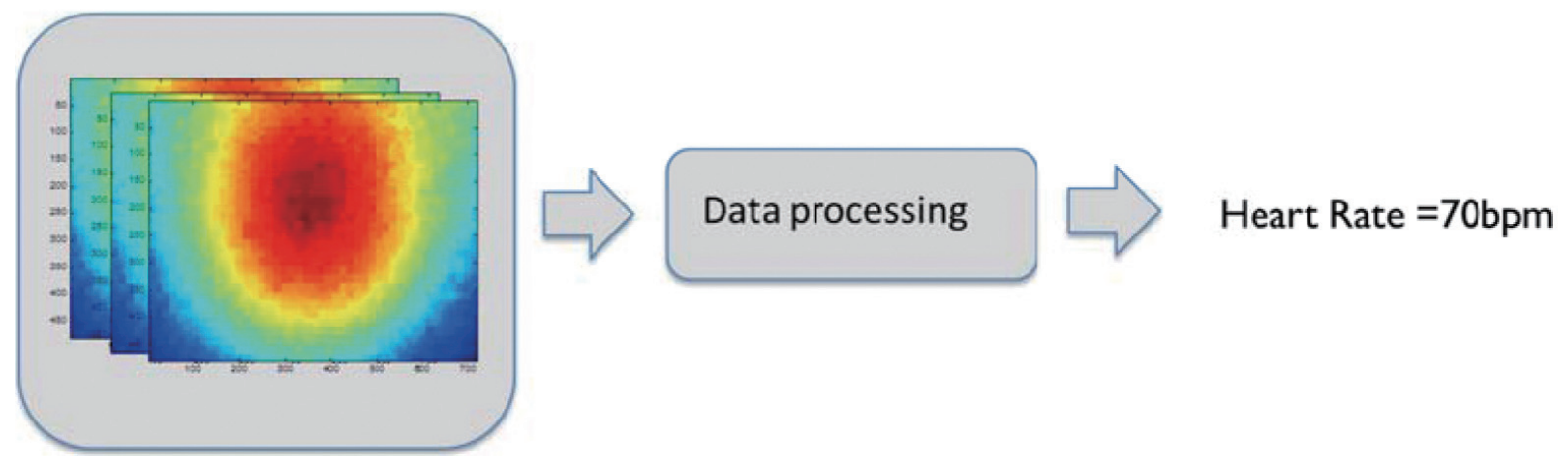

Fig. 9. Heart rate detection. (Colours are visible in the online version of the article; http://dx.doi.org/10.3233/TAD-150425) 


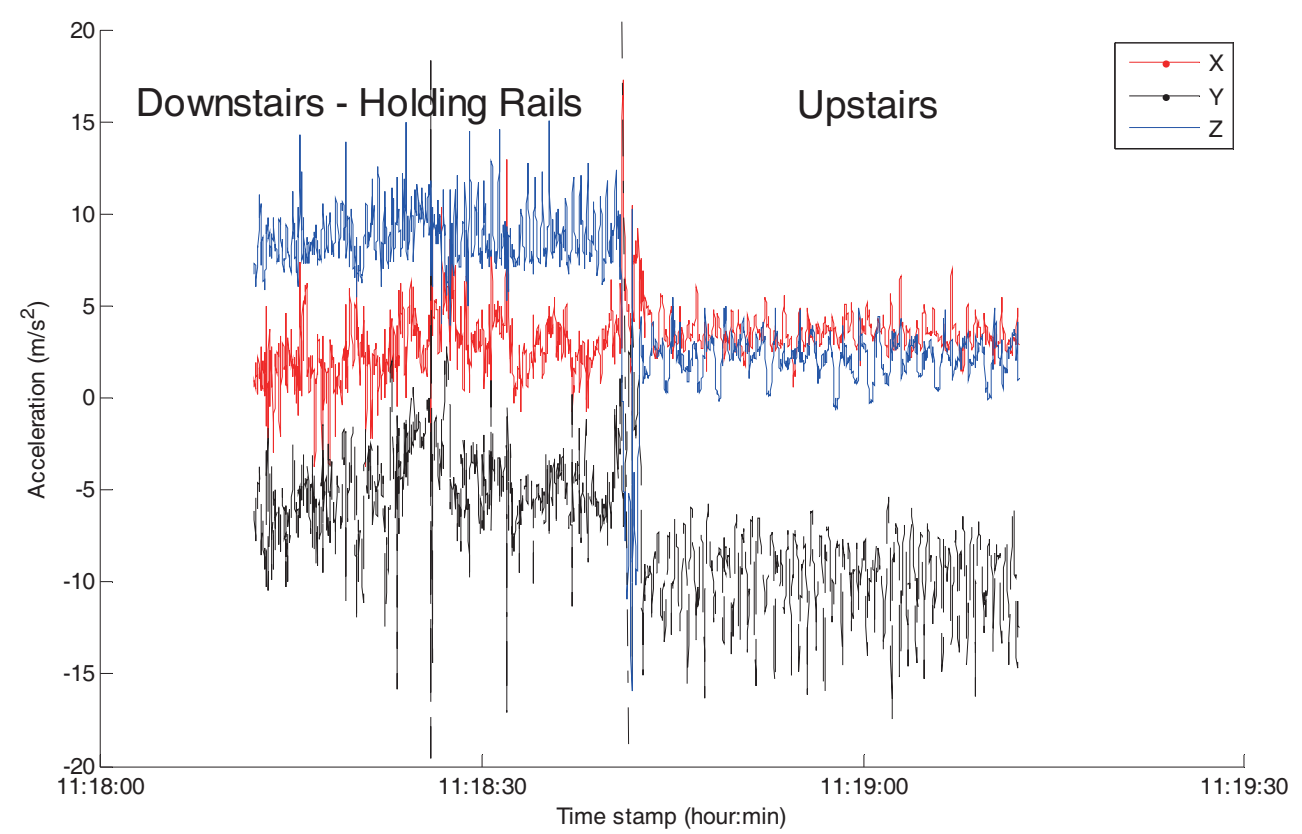

Fig. 10. Activity data recorded from the left hand whilst walking down the stairs holding the support rails (left) and walking up stairs without holding the rails. (Colours are visible in the online version of the article; http://dx.doi.org/10.3233/TAD-150425)

accuracy was typically \pm 1 beat per 15 seconds or \pm 4 beats per minute.

\section{Discussion}

The WWU used in our project measures physical activity parameters such as activity levels, step counts and device worn status, using the inbuilt accelerometer in the device. All of these parameters, when monitored over a period of time, can help to create a baseline for what is 'normal' for that person, and deviations from the baseline can be used to detect changes in the person's health, both physically and mentally. Studies have shown that the change in daily activity pattern over a period of time can even indicate the onset of cognitive impairment [36].

It could be extremely beneficial to monitor activities of an elderly person in an independent living situation where activity monitoring would typically form one part of a wider system. The other parameters that would be beneficial for the older person include postural transition [37], fall detection [38] and the classification or recognition of ADL [39]. It would be advantageous also to determine the ability of an older person to perform the different ADL.

This WWU offers an excellent and minimally intrusive way to monitor a person's well-being. However, it has to be noted that there are few limitations in our current approach, and the main ones are listed below:

All the parameters are measured from the accelerometer on the wrist, i.e., are based solely on the wrist movements. When the person is performing activities with a lot of wrist movement in the absence of movement in general, activity tends to be recorded as medium to high due to the use of the wrist as an indicator, rather than a recording of low activity as might be expected if a more holistic view were taken.

The step count detection is tested on accelerometer data of healthy young controls walking on a normal flat surface, walking up and down the stairs without holding the rails. Though, the step count detection performs well for healthy individuals, the algorithm has to be tested with a varied dataset. Figure 10 shows a walking pattern from the left wrist of a healthy subject, who was holding the support rails using his left hand whilst walking down the stairs. It can be seen clearly from the recorded data that these signals are very different to those captured whilst walking without a support (as shown in Fig. 7). Also, all the subjects in this study were healthy adults. So, this walking pattern could be significantly different for an older adult walking, particularly when walking with a support (such as a cane) and for people with a disability. The algorithm might require slight modifications to be adaptable for the different types of walking. Classifying the type of 
Table 2

Callisto 300 device specification

\begin{tabular}{ll}
\hline Device name & Callisto 300 \\
\hline Form factor & Watch \\
Dimension & $45 \mathrm{~mm} \times 42 \mathrm{~mm} \times 14 \mathrm{~mm}$ \\
Weight & $76 \mathrm{~g}$ \\
Operating system & Android 4.2 \\
Processor & MediaTek MT6572 @ 1.5 GHz dual core \\
Memory & $1 \mathrm{~GB}$ RAM \\
Removable storage & $8 \mathrm{~GB}$ \\
Connectivity & USB, Bluetooth and Wi-Fi \\
Battery & $600 \mathrm{mAh}$ \\
Camera & $3.0-5.0 \mathrm{M}$ \\
Screen & $40 \mathrm{~mm}, 240 \times 240$ pixels \\
Frequency ranges & GSM850/900/1,800 and 1,900(GPRS), SIM \\
\hline
\end{tabular}

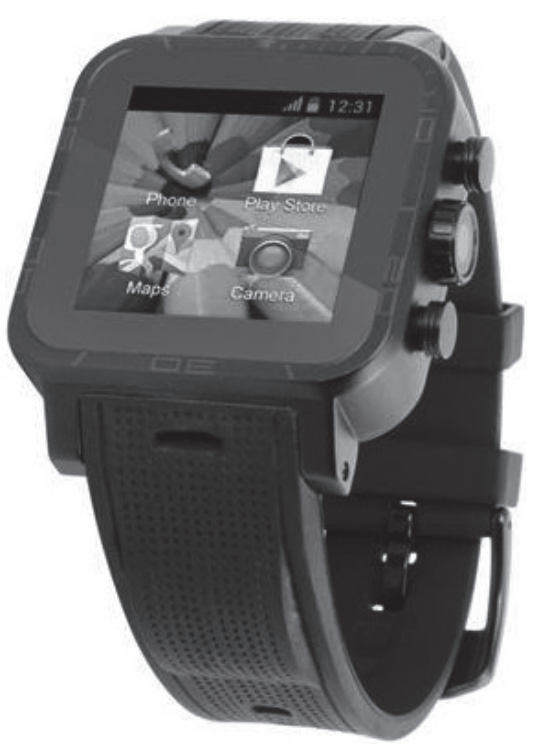

Fig. 11. The Callisto 300 Smartwatch.

walking and then performing the step count detection for that particular type of walking might be beneficial. Further work has to be performed to classify different walking patterns and activities, tailored to an individual person through an adaptive software algorithm.

The device worn status is also an important factor as the parameters that are monitored are based on the fact that the person is wearing the device all the time. When the person is not wearing the device, the parameters monitored are not valid. A limitation of this algorithm is that the device being worn could be identified incorrectly as device not being worn, for example, if the user is watching TV and sitting very still with the device on the wrist, the device could interpret the signal as if the device is not worn. Though we expect some minor movement in the accelerometer even when the user is still which is not seen when the device is not being worn, it will be useful to have an additional sen- sor (skin contact and temperature) for this purpose. As this device has only accelerometers for this purpose, further investigation needs to be performed on identifying the activity signature of removing the watch followed by a sedentary activity, to see if this can reduce false identification of the device not worn status.

The heart rate is recorded using the WWU camera which does not have a flash light. The presence of a flash light would be very useful while recording the heart rate as the light absorbed by the fingertip measured for this purpose. This is compensated by asking the subjects to record the camera data when facing a light source or in good ambient light. There is a possibility of inaccurate heart rate measurements in uncontrolled settings of the WWU camera, such as poor ambient lighting or presence of motion artefacts due to the movement of the finger tip of the subject. Whilst this is a limitation of the current hardware for the WWU it is expected that further iterations on the hardware will offer alternate solutions such as a camera flash, to provide better illumination or inbuilt heart-rate sensors. Other approaches in the data-analysis may also prove fruitful such as examining light in specific spectra or using other enhancement techniques.

An additional limitation with the current heart-rate monitoring solution is that of correct usage and compliance. Currently, the user is actively involved in the taking of the heart-rate data and need to be compliant with the required sensing regime. This is dependent on the intended use of the WWU and the context in which it is used, and likely to be limited to a few times per day. However, there is still the risk that measurements will not be taken or that they will be taken inaccurately due to device misuse. The only viable solution to this problem is to move to a device that has an on-board heart-rate sensor, such as the LG G WATCH R [40]. These are beginning to emerge onto the market and are 
expected to be adopted for future versions of the WWU hardware.

The current hardware used for the WWU is limited in battery life and offers between 5 and 8 hours when used continually. This can be extended by adopting an interval sampling technique where data is sampled for a period and then the device put into sleep mode for another period to spread the 'on' time throughout the day. This is recognised as a significant limitation of the hardware at this stage, but is present on all such devices. It should be noted that the Z1 was chosen over the other off-the-shelf devices as the openness of the device to connect and integrate with the other devices in the overall USEFIL system is more important for the project.

The hardware limitations, especially size and weight, on the WWU are mitigated to an extent by moving the app to new hardware as it becomes available. Due to the pace of technology development, a new device is being considered for the WWU hardware - the Callisto 300, shown in Fig. 11. The Callisto has a similar specification (shown in Table 2 for completeness) to the $\mathrm{Z} 1$, but is smaller and lighter $(45 \mathrm{~mm} \times 42 \mathrm{~mm} \times 14 \mathrm{~mm}$, $76 \mathrm{~g}$ ), has greater Wi-Fi range and is water resistant to the IP56 standard, potentially allowing use of the device whilst showering, although this is yet to be tested.

\section{Conclusions}

Activity monitoring has varied applications across healthcare and the wellness management of an older person. The assistive technologies available to perform activity monitoring could be wearable, environmental, or a combination of both. This paper presented the USEFIL system which is an assistive technology that combines both wearable sensors and environmental sensors to provide assistance in maintaining the independence of an older person.

Wearable intelligent systems using small, lightweight, high capacity platforms allow for high throughput of data for activity monitoring. The wrist wearable device which is part of the USEFIL system could also act as a stand-alone device to monitor the physical activity of a person. Through activity related measurements extracted from the WWU, much can be inferred about state of health or well-being of the person. The results to date look promising and could lay the foundation to developing a complex software algorithm that would help to identify and classify various activities.

We plan to improve the present activity monitoring system to include the classification of other typical ac- tivities for both young and older subjects. The ultimate aim is to create a robust software algorithm that could be run on a simple wrist worn device to monitor activity levels and falls, optimized especially for older people.

Further work on the WWU will focus on the expansion of the monitoring capabilities of the device to incorporate additional measures of activity such as ambulation type detection and postural change detection, as well as work on the existing algorithms to improve accuracy, particularly in regards to heart-rate. Testing of the device, both in terms of usability and functionality with different target groups will also form a key part of the ongoing work. Finally, the validation of the activity level calculations against gold standard calorimetry to compare calculated activity level with calorific expenditure will be undertaken.

\section{Acknowledgements}

This work was supported by USEFIL project, funded by the European Commission within the 7th Framework Program (Grant agreement no: 288532). The authors would like to acknowledge the input from the rest of the USEFIL team.

\section{References}

[1] World Health Organization. Health Topics Ageing [information page on the Internet]. 2014 [cited 2014 Dec 17] Available from: http://tinyurl.com/43qlkr9.

[2] Humphereys G. The Health-Care Challenges Posed by Population Ageing. Bull World Health Organ. 2012; 90(2).

[3] Wiles JL, Leibing A, Guberman M, Reeve J, Allen RES. The Meaning of "Ageing in Place" to Older People. Gerontologist. 2012; 52(3):357-366.

[4] Grguric A. ICT towards elderly independent living. Research and Development Centre, Ericsson Nikola Tesla. 2012. [cited 2014 Dec 17]. Available from: http://tinyurl.com/qxu2vb2.s

[5] Prince SA, Adamo KB, Hamel ME, Hardt J, Gorber SC, Tremblay M. A comparison of direct versus self-report measures for assessing physical activity in adults: a systematic review. Int J Behav Nutr Phys Act. 2008; 5. doi:10.1186/14795868-5-56.

[6] World Health Organization. Physical Activity and Older Adults: Recommended levels of physical activity for adults aged 65 and above [information page on the Internet]. 2014 [cited 2014 Dec 17] Available from: http://tinyurl.com/ o4js9d5.

[7] Shephard RJ. Limits to the measurement of habitual physical activity by questionnaires. Br J Sports Med. 2003; 37:197-206 doi:10.1136/bjsm.37.3.197.

[8] Berlin JE, Storti KL, Brach JS. Using Activity Monitors to Measure Physical Activity in Free-Living Conditions. J Am Phys Ther Assoc. 2006; 86:1137-1145. 
[9] Arcelus A, Jones MH, Goubran R, Knoefel F. Integration of Smart Home Technologies in a Health Monitoring System for the Elderly. In: Advanced Information Networking and Applications Workshops, 2007, AINAW '07. 21st International Conference on. (2):820-825.

[10] Godfrey A, Bourke AK, Olaighin GM, Van de Ven P, Nelson J. Activity classification using a single chest mounted tri-axial accelerometer. Med Eng Phys. 2011; 33:1127-1135.

[11] Song W, Ade C, Broxterman R, Nelson T, Warren S. Activity recognition in planetary navigation field tests using classification algorithms applied to accelerometer data. In: Engineering in Medicine and Biology Society (EMBC), 2012 Annual International Conference of the IEEE. 2012; 1586-1589. doi: 10.1109/EMBC.2012.6346247.

[12] Oshima Y, Kawaguchi K, Tanaka S, Ohkawara K, Hikihara Y, Ishikawa-Takata K, et al. Classifying household and locomotive activities using triaxial acceleration. Gait Posture. 2010; 31:370-374

[13] Xu M, Iyengar S, Goldfain A, Roy Chowdhury A, DelloStritto J. A two-stage real time activity monitoring system. In: Body Sensor Networks (BSN), 2011 International Conference on. 2011; 191-193. doi 10.1109/BSN.2011.31.

[14] Mathie MJ, Coster ACF, Lovell NH, Celler BG. Accelerometry: providing an integrated, practical method for long-term, ambulatory monitoring of human movement. Physiol Meas. 2004; 25(2):R1-20.

[15] Cleland I, Kikhia B, Nugent C, Boytsov A, Hallberg J, Synnes $\mathrm{K}$, et al. Optimal placement of accelerometers for the detection of everyday activities. Sensors. 2013; 13:9183-9200.

[16] Banos O, Damas M, Pomares H, Prieto A, Rojas I. Daily living activity recognition based on statistical feature quality group selection. Expert Syst Appl. 2012; 39:8013-8021.

[17] Chien C, Pottie GJ. A universal hybrid decision tree classifier design for human activity classification. In: Engineering in Medicine and Biology Society (EMBC), 2012 Annual International Conference of the IEEE. 2012; 1065-1068. doi 10.1109/EMBC.2012.6346118

[18] Murphy SL. Review of physical activity measurement using accelerometers in older adults: considerations for research design and conduct. Prev Med. 2009; 48:108-114.

[19] Najafi B, Aminian K, Loew F, Blanc Y, Robert PA. Measurement of Stand-Sit and Sit-Stand Transitions using a miniature Gyroscope and its application in fall risk evaluation in the elderly. IEEE Trans Biomed Eng. 2002; 49(8):843-851.

[20] Benzo R. Activity monitoring in chronic obstructive pulmonary disease. J Cardiopulm Rehabil Prev. 200; 29(6):341347.

[21] Haeuber E, Shaughnessy M, Forrester LW, Coleman KL, Macko RF. Accelerometer monitoring of home- and community-based ambulatory activity after stroke. Arch Phys Med Rehabil. 2004; 85(12):1997-2001.

[22] Kop WJ, Lyden A, Berlin AA, Ambrose K, Olsen C, Gracely $\mathrm{RH}$, Williams DA, Clauw DJ. Ambulatory monitoring of physical activity and symptoms in fibromyalgia and chronic fatigue syndrome. Arthritis Rheumatol. 2005; 52:296-303.

[23] Hong Y-J, Kim I-J, Ahn SC, Kim H-G. Mobile health monitoring system based on activity recognition using accelerometer. Simulation Modelling Practice and Theory. 2010; 18(4):446-455.

[24] USEFIL Consortium. Welcome to USEFIL [project home page on the Internet]. 2014 [cited 2014 Dec 17]. Available from: http:// www.usefil.eu/.

[25] ActiGraph, LLC. Actigraph [product home page on the Internet]. 2014 [cited 2014 Dec 17]. Available from: http://www. actigraphcorp.com/.

[26] Fitbit Inc. Fitbit [product home page on the Internet]. 2014 [cited 2014 Dec 17]. Available from: http://www.fitbit. com/uk.

[27] Jawbone. Up 24 [product home page on the internet]. 2014 [cited 2014 Dec 17]. Available from: http://jawbone.com/ store/buy/up24.

[28] Linuxslate.com. Linuxslate.com Independent Review Z1/ Rock Android 2.2 GSM Watch Phone [product review on the Internet]. 2012 [updated 20 July 2012, cited 2014 Dec 17]. Available from: http://tinyurl.com/ljsyaup.

[29] Amor JD, Detecting and monitoring behavioural change through personalised ambient monitoring. University of Southampton, Institute of Sound and Vibrarion Research, Doctoral Thesis. 2011.

[30] Easton C, Philip N, Aleksandravicius A, Pawlak J. Muggeridge DJ, Domene PA, et al. Validity of Smartphone Accelerometers for Assessing Energy Expenditure during Fast Running. In: XIII Mediterranean Conference on Medical and Biological Engineering and Computing 2013 IFMBE Proceedings. 2014; 41:1911-1914.

[31] Amor JD, Ahanathapillai V, James C. Detecting and analysing activity levels for the wrist wearable unit in the USEFIL project. In: XIII Mediterranean Conference on Medical and Biological Engineering and Computing 2013 IFMBE Proceedings. 2014; 41:1201-1204.

[32] Hatano Y. Use of the pedometer for promoting daily walking exercise. Intern Council for Health, Phys Educ, and Recreation. 1993; 29:4-8.

[33] Bravata DM, Smith-Spangler C, Sundaram V, Gienger AL, Lin N, Lewis R, et al. Using Pedometers to Increase Physical Activity and Improve Health: A Systematic Review. JAMA. 2007; 298:2296-2304.

[34] Leidy NK, Kimel M, Ajagbe L, Kim K, Hamilton A, Becker $\mathrm{K}$. Designing trials of behavioral interventions to increase physical activity in patients with copd: insights from the chronic disease literature. Respir Med. 2013; 108(3):472-481.

[35] Rustand A. Ambient-light photoplethysmography. Institutt for elektronikk og telekommunikasjon. Master's Thesis. 2012

[36] Noury N, Berenguer M, Teyssier H, Bouzid M-J, Giordani M. Building an Index of Human Activity from the activity on the Residential Electrical Power Line. IEEE Trans Inf Technol Biomed. 2011; 15(5):758-66.

[37] Ahanathapillai V, Amor JD, Tadeusiak M, James CJ. Wristworn accelerometer to detect postural transitions and walking patterns. In: XIII Mediterranean Conference on Medical and Biological Engineering and Computing 2013 IFMBE Proceedings. 2014; 41:1515-1518.

[38] Bourke AK, O'Brien JV, Lyons GM. Evaluation of a threshold-based tri-axial accelerometer fall detection algorithm. Gait Posture. 2007; 26:194-199.

[39] Parkka J. Ermes M, Korpipaa P, Mantyjarvi J, Peltola J, Korhonen I. Activity classification using realistic data from wearable sensors. IEEE Trans Inf Technol Biomed. 2006; 10(1):119,128.

[40] LG Electronics. G Watch R [product home page on the internet]. 2014 [cited 2014 Dec 19]. Available from: http://tinyurl. com/nwh9sh8. 